\title{
Temporal summation related to the nature of the proximal stimulus for the warmth sense*
}

\author{
LAWRENCE E. MARKS and JOSEPH C. STEVENS \\ John B. Pierce Foundation Laboratory and Yale University, New Haven, Connecticut 06519
}

\begin{abstract}
Magnitude estimations of the warmth aroused by radiant stimulation of the forehead showed that warmth obeys the psychophysical power law when the duration of the stimulus is relatively long ( 3 or 6 sec). When duration is short 10.25. 0.5 . or $1 \mathrm{sec}$ ). however, warmth grows as a more complex function of irradiation. The family of psychophysical functions measured for the various durations can be used to generate the rules by which radiant intensity and duration trade to preserve constant warmth. These rules vary systematically from complete temporal summation (i.e.. complete reciprocity) near threshold to less and less complete summation as warmth level increases. When, however. the stimulus is expressed as an equivalent change of temperature in the skin, there is no summation, only adaptation. It can be shown that temporal summation observed psychophysically must result from the heat-transfer properties of skin tissue.
\end{abstract}

Most, if not all, of the senses have the capacity to integrate the effects of stimulation over brief periods of time. the pervasive sensory property usually termed temporal summation. This paper asks how temporal summation works in the warmth sense, from near threshold to moderately warm levels.

There are two fundamental measures of temporal summation. One states how duration and intensity must covary to maintain a constant liminal or supraliminal sensation. The other states how duration influences the psychophysical relation between sensation magnitude, $\psi$, and stimulus intensity, $\varphi$. In many sense modalities, $\psi$ relates to $\varphi$ by a power function (S. S. Stevens, 1961)

$$
\psi=\mathrm{k} \varphi^{\beta} .
$$

Studies of vision and hearing (J. C. Stevens \& Hall, 1966) show that the sizes of the constants, $\beta$ and $k$, depend systematically on duration and can serve as useful indices of the temporal properties of the sense modality. The present study poses analogous questions about the nature and parameters of the psychophysical function for warmth.

Heretofore, the study of temporal summation of warmth has focused on the behavior of the absolute threshold (Geblewicz, 1935; Hendler, Hardy, \& Murgatroyd, 1963; J. C. Stevens, Okulicz, \& Marks, 1973). This work reveals that duration counts heavily in the first second of stimulation, but virtually not at all thereafter. Two studies (Geblewicz, 1937; Marks \& J. C. Stevens, 1968b) have touched cursorily on the question of how stimulus duration might influence sensory magnitude, but the picture is fragmentary.

In the search for larger perspective on temporal summation, the present study called on Ss to make magnitude estimations of the warmth aroused by irradiating the forehead for various durations and at

*This research was supported by Grant AFOSR 70-1950 from the United States Air Force Office of Scientific Research. various intensities. The resulting psychophysical functions serve to portray the summational properties of the warmth sense over most of its dynamic range. In brief, it turns out that when the stimulus is measured distally (i.e., as irradiance of the skin surface), the warmth sense displays nearly complete summation at low intensities and partial summation at high intensities. When, however, the stimulus is measured proximally (i.e., as temperature rise in the vicinity of warmth receptors), the warmth sense appears to display no summation at all. Hence, the key to summation seems to lie in the heat-conductive properties of the skin tissues.

\section{METHOD}

Apparatus

The apparatus consisted of a source of infrared irradiation (General Electric T-3 quartz lamp) mounted in a parabolic reflector, a shutter, and an aluminum shield having a rectangular aperture of $21.8 \mathrm{~cm}^{2}$. The $\mathrm{S}$ rested his forehead (blackened with India ink) against a cork lining on his side of the shield, thereby: exposing $21.8 \mathrm{~cm}^{2}$ of his skin to stimulation. Over this area, the density of radiant flux varied less than $10 \%$. The irradiance of the stimulus was measured with a Hardy radiometer (Hardy, Wolff, \& Goodell, 1952) and was controlled by adjusting the voltage to the lamp. Exposure duration was controlled with an electrical timer that operated the shutter. Air temperature was held constant at $21^{\circ} \mathrm{C}$, relative humidity at $30^{\circ} \mathrm{c}$.

\section{Procedure}

The task was magnitude estimation of warmth. The instructions called on the $S$ to assign to the first stimulus whatever number seemed most appropriate to stand for the warmth experienced at the end of exposure, and to succeeding stimuli to assign other numbers in proportion to warmth. Stimuli were given at the rate of one every $45 \mathrm{sec}$.

There were four experiments. Experiment I used 24 different stimuli: five levels of irradiance at each of four durations $(0.25$. $0.5,1$, and $3 \mathrm{sec})$ and four levels at a fifth duration $(6 \mathrm{sec})$. presented in a different random order to each $S$. After the first stimulus, which did not count, each stimulus was presented and judged twice in a session. (This same procedure was followed also in Experiments II-IV.) Fourteen men served as Ss. 


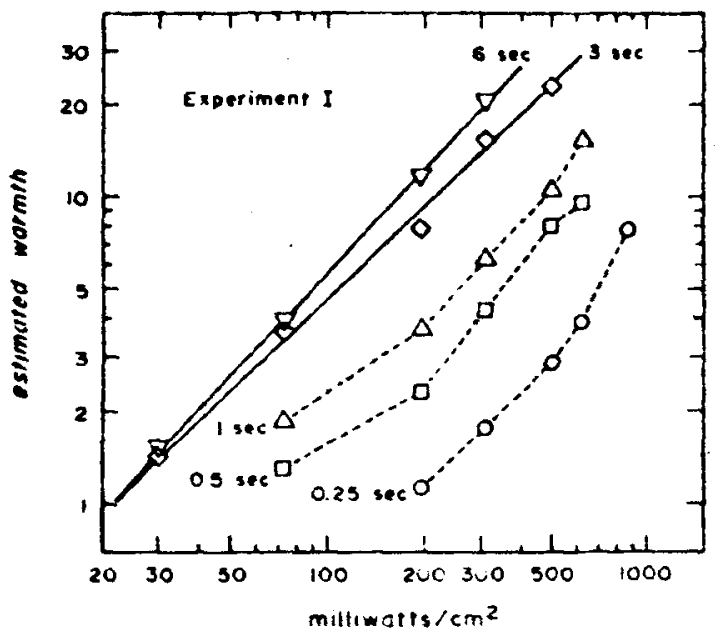

Fig. 1. Experiment I: Magnitude estimates of warmth as a function of irradiance of the forehead. The parameter is stimulus duration.

Fxperiment II explored a smaller range of durations in slightly more detail. There were six levels of irradiance at each of three durations $(0.25,0.5$, and $1 \mathrm{sec})$. Fourteen men served as $\mathrm{Ss}$.

Finally. Experiments III and IV examined one duration each: $1 \mathrm{sec}$ (III) and $3 \mathrm{sec}$ (IV). Fourteen men served in Experiment III. and 21 men and 3 women in Experiment IV.

\section{RESULTS AND DISCUSSION}

\section{Psychophysical Functions for Warmth}

Figure 1 shows the geometric means of the warmth estimates (Experiment I), plotted as functions of irradiance. The results for different durations appear displaced horizontally, from one another. That is because. given the same irradiance, the longer the duration. the greater the warmth. In other words, temporal summation operates over these ranges of stimulus irradiance and duration.

For 3-and 6-sec durations, power functions (straight lines in log-log coordinates) adequately describe the data, conforming to Eq. 1. But power functions are plainly not appropriate for the shorter durations, which vielded curvilinear (concave upward) functions.

This type of curvature is rarely encountered in psychophysical functions. Typical deviation from a simple power function is a downward concavity in the vicinity of the threshold (Marks \& J. C. Stevens, 1968a). This unexpected result provided the impetus to undertake Experiments II and III.

Figure 2 shows the geometric means of the warmth estimates (Experiments II and III) plotted as a function of irradiance. Again. upwardly concave functions plainly obtain at short durations.

Figure 3 brings together the results from the first three experiments. (The mean magnitude estimates for Experiment II were multiplied by 0.8 to bring them into alignment with those of the other experiments. This transformation does not affect the forms or slopes of the functions, but merely adjusts their positions vertically in the plane.) The psychophysical function for each short duration can be described well by two intersecting line segments. The sharp intersections drawn may not be real, i.e., the transitions from lower to upper segments could be more gradual; but the use of straight line segments is a convenience that does not do obvious injustice to the data. Note that all of the intersections of the two segments take place at approximately the same level of subjective warmth, $\psi \approx 2$.

The slopes (exponents) of the lower portions of the bisegmented functions all equal 0.7 . The slopes of the upper portions are: $1.3(0.25 \mathrm{sec}), 1.2(0.5 \mathrm{sec})$, and 1.0 ( $1 \mathrm{sec})$. Thus, the upper portion tends to steepen as duration shortens. The slopes of the unisegmented

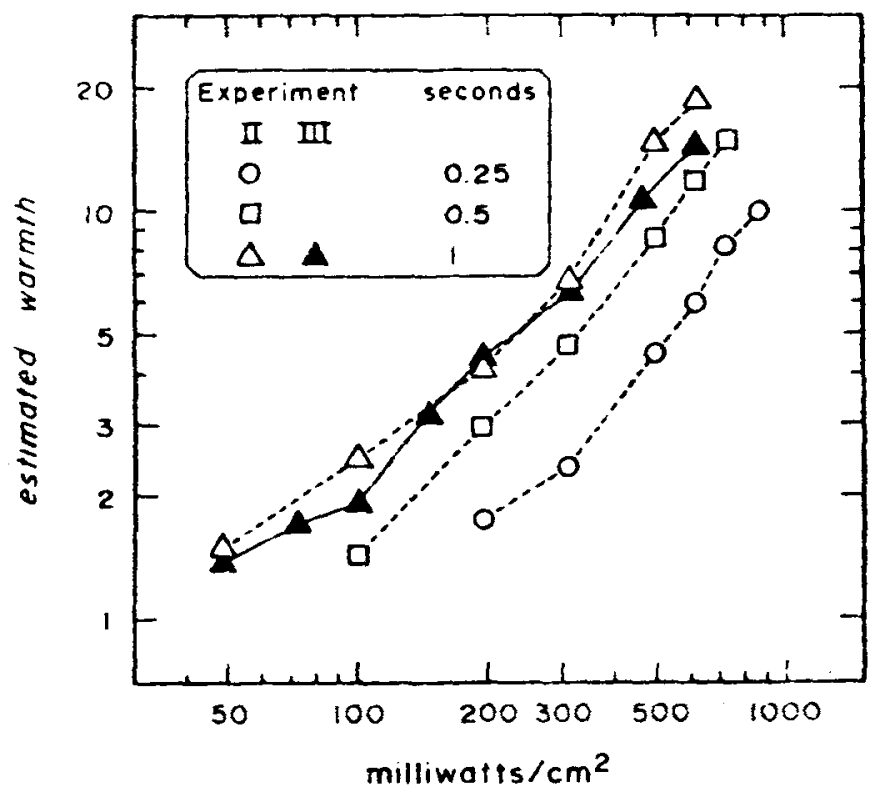

Fig. 2. Experiments II and III: Magnitude estimates of warmth as a function of irradiance of the forehead. The parameter is stimulus duration.

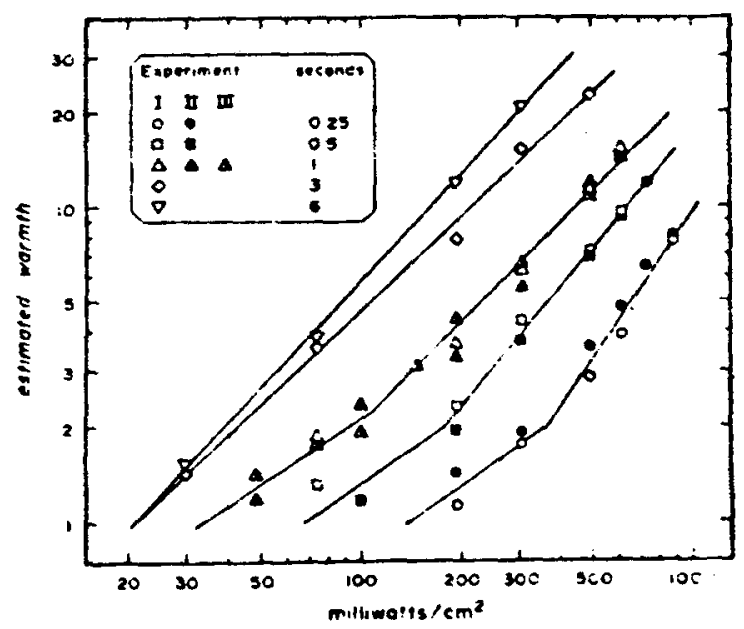

Fig. 3. Magnitude estimates of warmth from Experiments I. II, and III as functions of irradiance. 
functions equal $1.0(3 \mathrm{sec})$ and $1.1(6 \mathrm{sec})$.

Might there be some systematic, though smaller, deviation from the power function for longer durations? The large spacing between stimuli at durations of 3 and $6 \mathrm{sec}$ could easily have passed over small irregularities. Reexamination of earlier data obtained with $3-\mathrm{sec}$ stimulation (J. C. Stevens \& Marks, 1971) gave evidence of an irregularity. Experiment IV examined in detail the warmth function for $3-\mathrm{sec}$ stimulation. Figure 4 compares results of Experiment IV with those of the earlier experiment by Stevens and Marks (also for stimulation of $21.8 \mathrm{~cm}^{2}$ of the forehead). The similarity is strong. An irregularity appears at about $50 \mathrm{~mW} / \mathrm{cm}^{2}$. but it is small, covering a range of about $10 \mathrm{~mW} / \mathrm{cm}^{2}$. This deviation from a simple power function is minor compared with the "discontinuities" obtained with shorter exposure durations (see Fig. 3). We may also ask at what level of warmth the discontinuity takes place. The answer lies in Fig. 3, which shows that the discontinuity seems always to occur at the same level of warmth! That figure shows that a stimulus of $50 \mathrm{~mW} / \mathrm{cm}^{2}$ presented for $3 \mathrm{sec}$ (the region of discontinuity in Fig. 4) produced warmth equal to 2.3 . This is practically the same warmth at which the other discontinuities appear.

\section{Warmth as a Function of Duration}

The data of Fig. 3 are replotted in Fig. 5 to show how

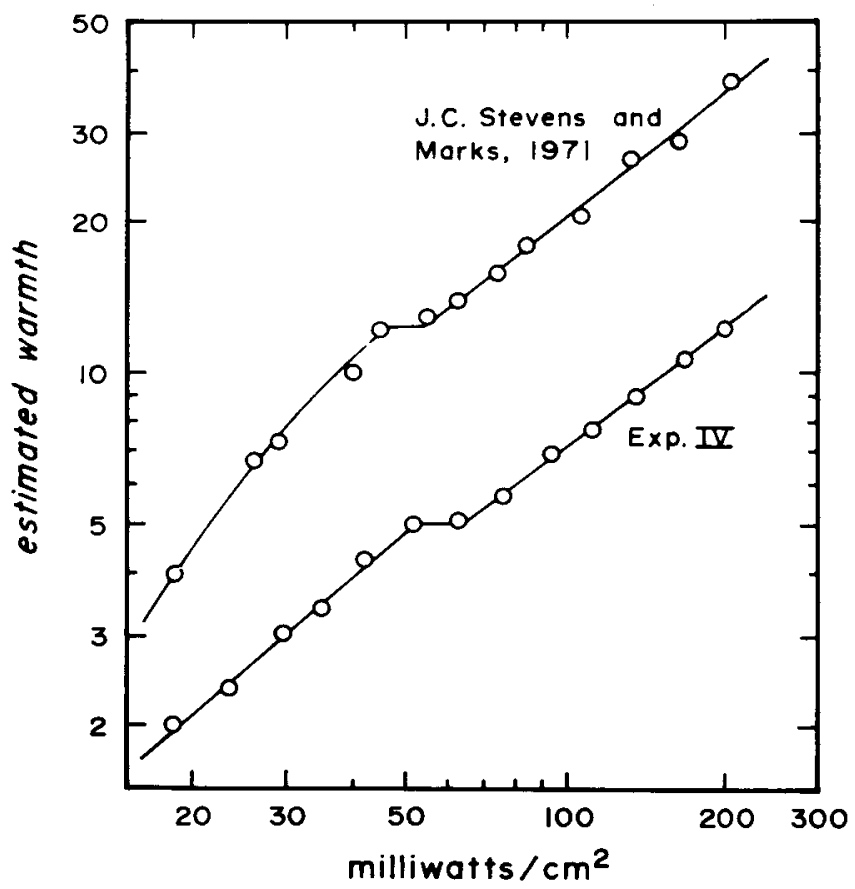

Fig. 4. Magnitude estimates of warmth as a function of irradiance. Stimulus duration of $3 \mathrm{sec}$ (Experiment IV). Also shown are results obtained by J. C. Stevens \& Marks (1971) with the same stimulus duration and area. The latter have been displaced upward in order to improve clarity.

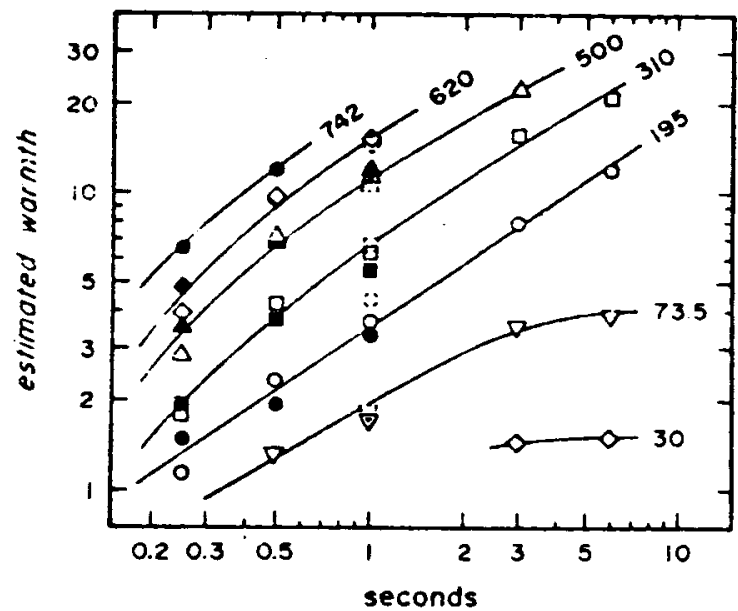

Fig. 5. Magnitude estimates of warmth (Experiments I-III). plotted as a function of stimulus duration. The parameter is irradiance in $\mathrm{mW} / \mathrm{cm}^{2}$.

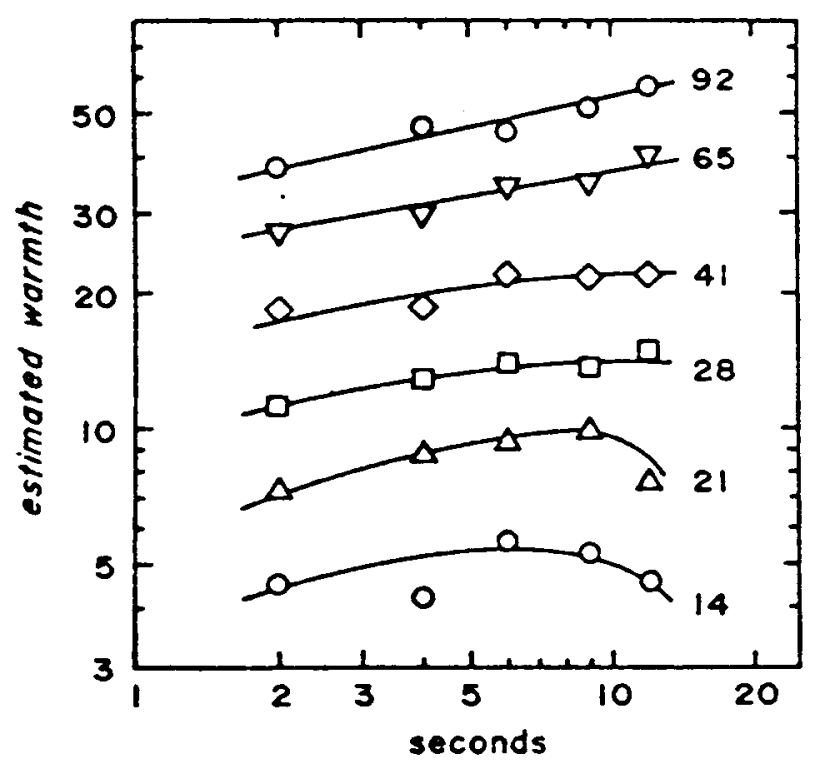

Fig. 6. Magnitude estimates of warmth as a function of stimulus duration (from Marks \& J. C. Stevens, 1968b). Here the whole front surface of the body was stimulated. The parameter is irradiance in $\mathrm{mW} / \mathrm{cm}^{2}$.

warmth grows with stimulus duration under constant level of irradiation. For all levels of irradiation except perhaps the lowest, warmth grows continuously with duration. For comparison, Fig. 6 shows how warmth changes over time when the whole front surface of the body is stimulated (Marks \& J. C. Stevens, 1968b). Because the warmth sense displays extensive spatial summation, stimulation of so large an area required relatively low levels of irradiance. The striking result was that warmth varied so little with duration. At the highest irradiances ( 65 and $92 \mathrm{~mW} / \mathrm{cm}^{2}$ ), warmth did increase continuously, but at lower levels (28 and $\left.41 \mathrm{~mW} / \mathrm{cm}^{2}\right)$, it remained virtually constant after about $4 \mathrm{sec}$. That outcome agrees well with the present one, namely that 


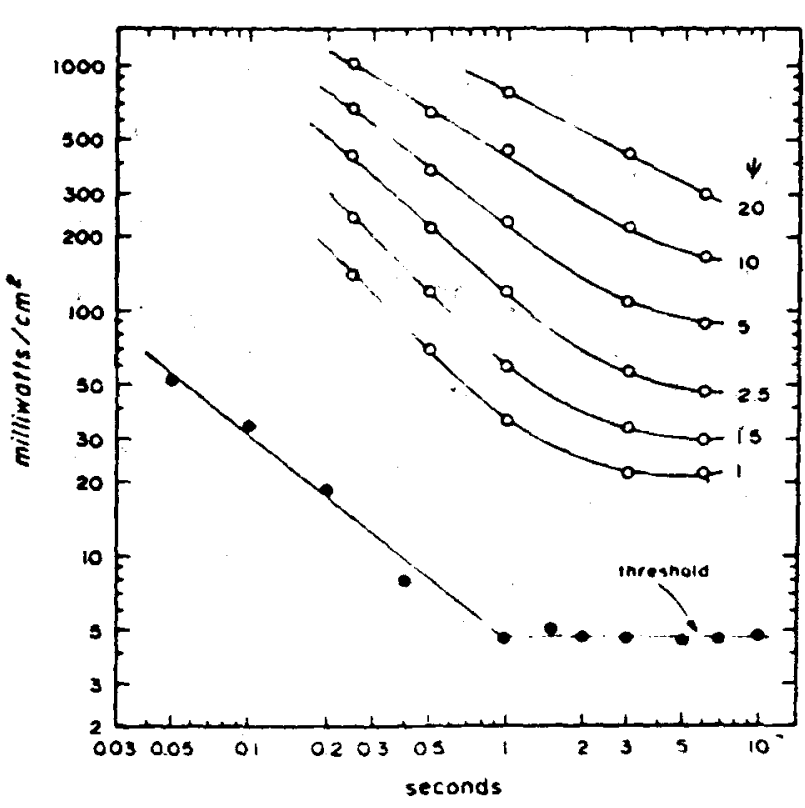

Fig. 7. Equal-sensation contours showing how raalant intensity and duration covary to produce various constant levels of warmth. The filled circles are thresholds measured by J. C. Stevens, Okulicz, and Marks (in press) for the same $21.8 \mathrm{~cm}^{2}$ area of the forehead.

at 30 and $73.5 \mathrm{~mW} / \mathrm{cm}^{2}$ warmth did not change after $3 \mathrm{sec}$ (Fig. 5). Thus. when stimulus irradiance is low, warmth seems to show little growth over time.

\section{Intensity-Time Reciprocity in the Warmth Sense}

The psychophysical functions of Fig. 3 contain the information needed to calculate how irradiance and duration must covary to produce any constant level of warmth sensation. This is done by making a horizontal cut through the functions at any chosen ordinate, $\psi$. Figure 7 shows a family of equal-warmth trading functions generated by this procedure. Given a low constant degree of warmth $(\psi=1$ or 1.5$)$, there is a near perfect time-intensity reciprocity over the first second or so (i.e., the trading function has a slope of about -1.0 ). Beyond a second or so, the degree of summation declines ultimately to zero. This is indicated in Fig. 7 as a gradual approach of the trading function to the horizontal: the data do not rule out, however, the possibility that the transition from summation to no summation might be abrupt (i.e., that there is a "critical duration"). This is the way the absolute threshold behaves. Threshold measurements for stimulation of $21.8 \mathrm{~cm}^{2}$ of the blackened forehead with pulses of infrared radiation (J. C. Stevens, Okulicz, \& Marks, 1973) are shown as the filled circles in Fig. 7. The threshold function has a slope of -0.8 . This result-slightly more summation just above than at threshold-has a counterpart in the temporal summation of sound energy by the ear (J.C. Stevens \& Hall, 1966).

As the criterial level of warmth increases. the slope of the trading functions gradually flattens, indicating a steady diminution of the degree of summation. At the same time, however, summation tends to extend to longer and longer durations. At $\dot{\psi}=10$ and 20 . for example. almost the entire range of duration-from 0.25 to $6 \mathrm{sec}-\mathrm{can}$ be described by single straight lines. These lines have slopes of about -0.5 . Thus, the degree of summation diminishes from complete reciprocity at low warmth to square-root summation at higher levels. (The contour, $\psi=10$, corresponds to what can best be called "moderate" warmth. The stimulus that produces it is about one-fourth that needed to produce threshold pain.)

\section{Temporal Summation Related to Temperature Changes at the Skin Surface}

From a psychophysical point of view, the warmth sense, like the other senses, displays temporal summation over its dynamic range; but a different picture can emerge if the stimulus is viewed proximally. In vision, the proximal stimulus may be viewed as the number of decomposed molecules of photopigment. Since this quantity is proportional to energy (the product of intensity and duration), the issue of temporal summation resolves itself. Similarly, temporal summation of warmth appears in a new light when the stimulus is specified in terms of changes in temperature of the skin tissue.

The lengthening "critical duration" with increased warmth level stands in contrast to a shortening visual critical duration with increased brightness. In any case, the long critical durations measured here demonstrate that the warmth sense has a sluggish temporal response. This sluggishness shows itself also in poor temporal resolution, as measured in terms of the thermal analogue of CFF. The skin is unable to resolve radiant pulses delivered at rates much faster than $34 / \mathrm{sec}$ (Herget. Granath, \& Hardy, 1941).

Under the conditions of the present experiments (Ss sedentary and stimulus durations shorter than $10-15 \mathrm{sec})$, the skin responds passively to thermal stimulation. This makes it possible to estimate the changes in skin temperature at any depth produced by irradiation of the surface, using a model provided by Stolwijk \& Hardy (1965) (see also Marks \& J. C. Stevens, 1968b). Such temperature changes were calculated by simultaneous integration of a set of eight differential equations. Each equation accounts for heat flow into and out of a particular layer of the skin. Because the Ss' foreheads were blackened, it was assumed that all of the incident radiation was absorbed in the most superficial layer. The simulation provides estimates of temperature changes at the skin surface (depth, $0.025 \mathrm{~mm}$ ) and at depths of $0.075 .0 .125,0.175,0.3,0.5,0.7$ and $5.8 \mathrm{~mm}$ below the surface.

Figure 8 shows how the constant warmth functions look when the stimulus is reckoned as the terminal 
change in superficial skin temperature $(\Delta T)$ predicted by the Stolwijk-Hardy model (each combination of irradiance and duration was entered into the model, and the resulting changes in skin temperature were computed). Over short durations, the contours show only partial summation, even at low levels of warmth. Again, as level increases, degree of summation declines. And, over long durations, there is no summation at all. At low levels of warmth, the "reverse" of summation obtains; i.e., greater changes in temperature are required at longer durations to produce constant warmth. As level of warmth increases, the degree of this "reverse summation" diminishes, so that at the highest levels ( $\psi=$ 10 and 20), the contours are virtually horizontal. At these high levels, warmth varies only with superficial skin temperature and not at all with duration.

This more complex picture of temporal dependence arises because change in skin temperature $(\Delta T)$ is directly proportional to level of irradiance, but proportional to the square root of duration. The square-root relation obtains whenever all of the incident radiation is absorbed near the skin surface, as it is when the skin is blackened (Buettner, 1951).

\section{Temporal Summation Related to Temperature Change Within the Skin}

Although the location of warmth receptors is not known, it is taken for granted that they do not lie right at the surface. A depth of approximately $0.2 \mathrm{~mm}$ might be a good possibility: at least so suggested Hendler, Hardy. and Murgatroyd (1963), who showed that $\Delta \mathrm{T}$ for absolute threshold is virtually constant $0.2 \mathrm{~mm}$ below the surface. Figure 9 shows how the trading functions look when stimulus magnitude is considered to be $\Delta \mathrm{T}$ at $0.175 \mathrm{~mm}$ below the surface. Over the first second or so, low-level warmth does not depend on duration (same result as Hendler et al obtained), but high-level warmth

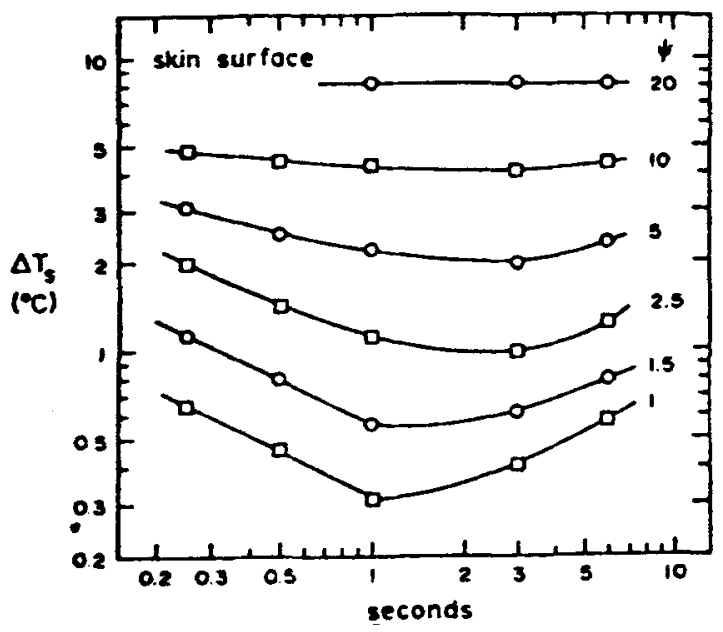

Fig. 8. Equal-sensation contours showing how rise in superficial skin temperature $(\Delta T)$ and the time taken to yield that rise covary to produce various constant levels of warmth.

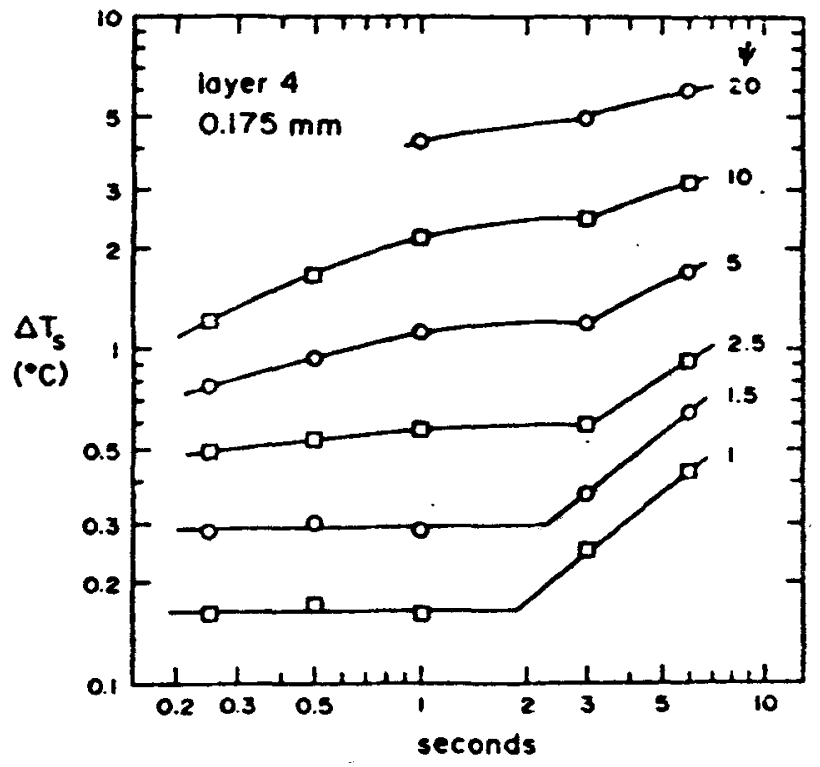

Fig. 9, Equal-sensation contours showing how rise in temperature $(\Delta T) 0.175 \mathrm{~mm}$ below the skin surface and the time taken to yield that rise covary to produce various constant levels of warmth.

apparently does. The difference between outcomes at low and at high warmth reflects the fact that the psychophysical functions measured with different constant stimulus durations (Fig. 3) are not all parallel to one another. If warmth were a function of $\Delta \mathrm{T}$, then all of the psychophysical functions in Fig. 3 would be parallel. This is so because, given any constant stimulus duration, $\Delta \mathrm{T}$ is directly proportional to irradiance. Because the psychophysical functions of Fig. 3 are not all parallel, it is not surprising that the trading functions of Fig. 9 are not all horizontal, even over short durations. Over long durations, Fig. 9, like Fig. 8, shows "reverse summation," but in Fig. 9 this shows up at all levels of warmth.

It is instructive to look also at a slightly deeper layer of skin: $0.3 \mathrm{~mm}$ below the surface. At this depth, the trading functions (Fig. 10) show a complete absence of summation. Instead, "reverse summation" becomes the rule; i.e., the longer the stimulus duration, the greater must be the change in temperature. It appears that at no depth in the skin does $\Delta \mathrm{T}$ provide a completely consistent correlate to warmth sensation, not even over durations shorter than $1 \mathrm{sec}$. The same conclusions hold even under modification of the model for skin temperature. Substantial changes can be made in the constants of the heat-flow equations without altering the general pictures shown in Figs. 8-10.

\section{Proximal Stimulus for Warmth Sesnation}

An earlier paper (Marks \& J. C. Stevens, 1968b) reported how warmth varies over time when a large portion of the body is irradiated. Warmth failed to correlate well with change in skin temperature $(\Delta T)$, 


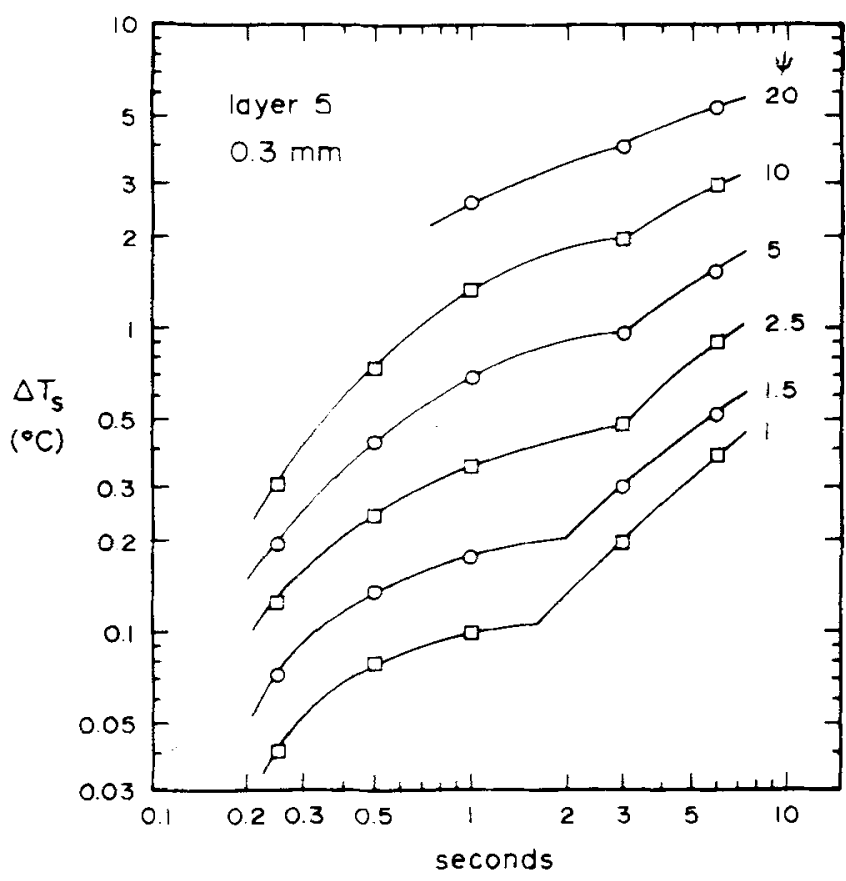

Fig. 10. Equal-sensation contours showing how rise in temperature $(\Delta T) 0.3 \mathrm{~mm}$ below the skin surface and the time taken to yield that rise covary to produce various constant levels of warmth.

with rate of change of skin temperature $(\mathrm{dT} / \mathrm{dt})$, or with local spatial gradient of temperature $(\mathrm{dT} / \mathrm{dx})$. Two possible correlates did emerge: (1) the difference between temperatures at two distinct depths in the skin and (2) $\Delta T$. coupled with some inhibitory process like adaptation that would mitigate the excitatory effects of continuously increasing $\Delta \mathrm{T}$ over time.

\section{Temperature Difference}

That warmth might depend on the difference between two temperatures was proposed in a qualitative manner by Lele, Weddell, \& Williams (1954), who postulated that warmth receptors might operate more like a bolometer than like a thermometer. Hendler, Hardy, and Murgatroyd (1963) attempted to specify the depths of the two critical layers. Using their measurements of warmth thresholds for both infrared and microwave radiation, they estimated the layers to lie 0.2 and $1.0 \mathrm{~mm}$ below the skin surface.

The present data do not square with a simple model of temperature difference. The trouble with the model lies in its basic linearity. Given any constant stimulus duration, tissue temperature at any depth increases in proportion to irradiance. It follows that the difference between temperatures at any two points in the skin must also increase in proportion to irradiance. If warmth were proportional to temperature difference, then the psychophysical functions obtained at different constant durations should all be parallel to one another. But Fig. 3 suggests that they are not. Therefore, it appears that temperature difference alone cannot predict warmth. However. it might be possible to rescue the model by postulating that warmth sensation depends on the difference between some nonlinear functions of two temperatures [i.e., on $F_{1}\left(T_{1}\right)-F_{2}\left(T_{2}\right)$ rather than on $\left.\mathrm{F}\left(\mathrm{T}_{1}-\mathrm{T}_{2}\right)\right]$.

\section{Adaptation}

The most that can be said is that the present results are not inconsistent with a hypothesis of adaptation, since no quantitative restrictions have been set on a model that incorporates adaptation. Eijkman and Vendrik's (1961) measurements of warmth thresholds obtained with microwave stimulation led them to postulate two adaptational mechanisms. The present results accord with that hypothesis: The discontinuities displayed in the $\Delta$ T-duration functions (Figs. 9 and 10) imply the operation of two mechanisms; the discontinuities and inflections in the psychophysical functions for warmth (Figs. 3 and 4), if they are real, may also be interpreted to reflect the transfer of mediation from one mechanism to another. Studies on spatial summation of warmth (J. C. Stevens \& Marks, 1971; Marks, 1971) led us to speculate that the level-dependent change in degree of spatial summation might be the product of transfer from one mechanism to another. Warmth may depend, therefore, on the activity of two mechanisms-perhaps two different populations of receptors-that differ both with respect to their spatial properties (degree of spatial summation) and with respect to their temporal properties (rates and/or extents of adaptation).

\section{REFERENCES}

Buettner, K. Effects of extreme heat and cold on human skin. I. Analysis of temperature changes caused by different kinds of heat application. Journal of Applied Physiology. 1951, 12 , 691-702.

Eijkman, E., \& Vendrik. A. J. H. Dynamic behavior of the warmth sense organ. Journal of Experimental Psychology, $1961,62,403-408$.

Geblewvicz, E. La relation du temps daction liminaire avec l'intensité pour les stimulations thermiques. Comptes Rendus de la Société de Biologie, 1935. 118.748-750.

Geblewicz. E. L'établissement de la sensation thermique en fonction de la durée d'excitation. Comptes Rendus de la Société de Biologie, 1937, 125,912-915.

Hardy, J. D.. Wolff, H. G.. \& Goodell. H. Pain sensations and reactions. Baltimore: Williams \& Wilkins. 1952.

Hendler, E.. Hardy, J. D., \& Murgatroyd, D. Skin heating and temperature sensation produced by infrared and microwave irradiation. In C. M. Herzfeld (Ed.), Temperoture: Its measurement and control in science and industry. Vol. 3. Part 3. New York: Reinhold, 1963. Pp. 211-230.

Herget, C. M.. Granath. L. P.. \& Hardy. J. D. Thermal sensation and discrimination in relation to intensity of stimulus. American Journal of Physiology, 1941, 134. 645-655.

Lele, P. P.. Weddell, G.. \& Williams, C. M. The relationship between heat transfer. skin temperature and cutaneous sensibility. Journal of Physiology. 1954. 126. 206-234. 
Marks. L. E. Spatial summation in relation to the dynamics of warmth sensation. International Journal of Biometeorology: $1971,15,106-110$.

Marks. L. E., \& Stevens. J. C. The form of the psychophysical function near threshold. Perception \& Psychophysics, 1968a. 4. 315.318 .

Marks. L. E.. \& Stevens, J. C. Perceived warmth and skin temperature as functions of the duration and level of thermal irradiation. Perception \& Psychophysics. 1968b. 4. 220-228.

Stevens. J. C., \& Hall. J. W. Brightness and loudness as functions of stimulus duration. Perception \& Psychophysics, 1966, 1, 319-327.

Stevens. J. C.. \& Marks, L. E. Spatial summation and the dynamics of warmth sensation. Perception \& Psychophysics, $1971,9,391-398$.
Stevens, J. C., Okulicz, W. C., \& Marks, L. E. Temporal summation at the warmth threshold. Perception \& Psychophysics, 1973, in press.

Stevens, S. S. The psychophysics of sensory function. In W. A. Rosenblith (Ed.). Sensory communication. New York: Wiley, 1961. Pp. 1-33.

Stolwijk. J. A. J.. \& Hardy. J. D. Skin and subcutaneous temperature changes during exposure to intense thermal radiation. Journal of Applied Physiology, 1965, 20, 1006-1013.

(Received for publication June 6, 1973; revision received July $19,1973$. ) 